\title{
Low LPA gene kringle IV-2 repeat copy number association with elevated lipoprotein (a) concentration as an independent risk factor of coronary atherosclerotic heart disease in the Chinese Han population
}

Lishan Sun, Ming Zong, Cuncun Chen, Lihong Xie, Fei Wu, Ming Yu and Lieying Fan*

\begin{abstract}
Background: Lipoprotein (a) [LP(a)], which is genetically determined by the LPA gene kringle IV type 2 (KIV-2) repeat copy number, has previously been reported in different populations. However, it is uncertain if the same occurs in the Chinese Han population. This study explored the correlation of Lp(a) mass or particle concentration with KIV-2 repeat copy number and application for coronary atherosclerotic heart disease (CAHD) risk assessment.

Methods: A cross-sectional study including 884 subjects was conducted. The Lp(a) level and routine risk factors of CAHD were compared. The KIV-2 copy number distribution, relationship with $L p(a)$, and assessment for CAHD risk were explored.

Results: The mean of $L p(a)$ mass or particle concentration in the CAHD group was higher than that in the nonCAHD group, while the KIV-2 copy number in the CAHD group was lower. Lp(a) had auxiliary values in gauging the type of plaque and was significantly higher in the soft-plaque group than that in the other two groups (200 mg/L [21.5 nmol/L], $166 \mathrm{mg} / \mathrm{L}[18.6 \mathrm{nmol} / \mathrm{L}], 149 \mathrm{mg} / \mathrm{L}[17.1 \mathrm{nmol} / \mathrm{L}]$, respectively, $P<0.05)$. Kappa test indicated divergence for the same individual using two $L p(a)$ concentrations (kappa value was $0.536[<0.75]$ ). Elevated Lp(a) was an independent CAHD risk factor, whatever mass or particle concentration, and large KIV-2 copy number was a protective factor.
\end{abstract}

Conclusion: Lp(a) level and small KIV-2 copy number are risk factors for CAHD in the Chinese Han population; furthermore, elevated $L p(a)$ may gauge the type of coronary plaque.

Keywords: Lipoprotein(a), Atherosclerotic heart disease, Kringle IV type 2 repeat

\footnotetext{
* Correspondence: flieying@yeah.net

Department of Clinical Laboratory, Shanghai East Hospital, Tongji University

School of Medicine, No. 150, Jimo Road, Shanghai 200120, People's Republic

of China
}

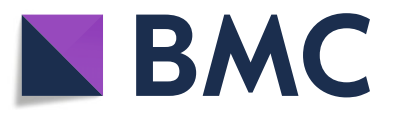

() The Author(s). 2018 Open Access This article is distributed under the terms of the Creative Commons Attribution 4.0 International License (http://creativecommons.org/licenses/by/4.0/), which permits unrestricted use, distribution, and reproduction in any medium, provided you give appropriate credit to the original author(s) and the source, provide a link to the Creative Commons license, and indicate if changes were made. The Creative Commons Public Domain Dedication waiver (http://creativecommons.org/publicdomain/zero/1.0/) applies to the data made available in this article, unless otherwise stated. 


\section{Background}

Several studies have confirmed that there is a close relationship between high lipoprotein (a) $[\mathrm{Lp}(\mathrm{a})]$ level and coronary atherosclerotic heart disease (CAHD) [1, 2]. These studies have also provided three levels of evidence. In the first level, a large number of case-control studies, cohort studies and large-sample-size epidemiological surveys as well as clinical meta-analyses have confirmed that there is a close relationship between individual $L p(a)$ level elevation and CAHD risk, cardiovascular event probability elevation and poor prognosis [3-6]. Evidence at the second level is based on large-samplesize pan-genomics studies, which correlate "single nucleotide polymorphism (SNP) and repeat copy number variation (CNV) in LPA gene" with "Lp(a) levels and CAHD occurrence." They These studies also provide direct genetic evidence about the relationship between high Lp(a) level and CAHD [7-9]. At the third level, evidence is based on the results of Mendelian randomized studies confirming that $\mathrm{Lp}$ (a) levels have a genetic predisposition in a given population, i.e., most of the Lp(a) determinant levels result from LPA gene mutations [10-12]. However, since a therapeutic regimen for effectively lowering $\mathrm{Lp}(\mathrm{a})$ is not currently available, direct evidence of lowering CAHD risk by lowering Lp(a) levels has not yet been determined detected in clinical practice. Whether patients with high $\mathrm{Lp}(\mathrm{a})$ need to receive treatment for lowering $\operatorname{Lp}(\mathrm{a})$ is still controversial, but many therapeutic clinical trials are underway [13-15]. The molecular structure of $\mathrm{Lp}(\mathrm{a})$ is similar to that of LDL and is composed of specific apo (a) molecules and LDL-like particles attached via a disulfide bond. The physiological functions of $\operatorname{Lp}(\mathrm{a})$ are unclear [16], and whether $\mathrm{Lp}(\mathrm{a})$ is an independent risk factor for CAHD has not been fully elucidated. There are two expression pathways for detecting $\mathrm{Lp}(\mathrm{a})$-related results: mass concentration and particle concentration (briefly, particle concentration). This issue further increases clinical study complexity with uncertain results [17]. Additionally, large-sample-size studies were recently conducted for exploring the clinical value of particle concentration but were inconclusive [18]. There is scant study on how LPA gene's kringle IV-2 (KIV-2) repeat copy number variation is distributed and affects $\operatorname{Lp}(\mathrm{a})$ concentration along with CAHD risk factor in the Chinese population [19, 20]. This study compared the difference between CAHD risk assessment by using $\mathrm{Lp}(\mathrm{a})$ mass concentration and particle concentration, and then established a method to measure the KIV-2 repeat copy number and explore the distribution in the Chinese Han population, the relationship with $\operatorname{lp}(\mathrm{a})$ concentration as well as risk assessment for CAHD.

\section{Methods}

\section{Study subjects}

The subjects were selected from patients who visited the Department of Cardiology in Shanghai East Hospital from October 2013 to January 2017. These patients had undergone coronary arterial computerized tomography angiography (CTA) for suspected coronary artery disease. Patients receiving nicotinic acid were excluded from this study. All 884 patients enrolled were of Chinese Han ethnicity including 456 males (51.6\%) and 428 females (48.4\%), with an average age of $59 \pm 9$ years. The experimental protocol was reviewed and approved by the Hospital Ethics Committee, and all enrolled subjects signed informed consents.

The coronary arterial CTA examination results were independently gauged and determined by two deputy chief physicians or similar level staff members. Clinical data of patients were then recorded, including gender, age, coronary arterial CTA examination result, etc. All subjects were divided into two CAHD and non-CAHD groups depending on coronary arterial CTA results and whether coronary atherosclerotic plaque was detected. Furthermore, based on the CT value (Hounsfield unit, $\mathrm{HU})$ of coronary atherosclerotic plaque in the stenotic position, the patients in the CAHD group were divided into 3 subgroups: soft plaque $(<60 \mathrm{HU})$, calcified plaque (>130 HU) and mixed plaque $(60 \sim 130 \mathrm{HU})$. The criteria for dividing subgroups were as follows. For patients who had coronary atherosclerotic plaque in various coronary artery branches and trunks, a patient with soft plaque in one or more locations was included in the soft-plaque subgroup; a patient with calcified plaque in one or more locations was included in the calcifiedplaque subgroup; a patient with no soft plaque or with only a mixed plaque (or more), or with both a mixed plaque (or more) and a calcified plaque (or more), was included into the mixed-plaque subgroup.

\section{Conventional clinical and laboratory indicator tests}

Subjects had to fast for more than $8 \mathrm{~h}$ before coronary arterial CTA examination. Venous blood was collected, and the samples were centrifuged at $1880 \times \mathrm{g}$ for $10 \mathrm{~min}$. Sera were separated for conventional biochemical tests, including $\mathrm{Lp}(\mathrm{a})$ particle concentration, $\mathrm{Lp}(\mathrm{a})$ mass concentration, triglycerides, total cholesterol, high density lipoprotein cholesterol (HDL-C), low density lipoprotein-cholesterol (LDL-C), and apolipoprotein B (Apo(B)); hypersensitive C-reactive protein (hs-CRP) and non-HDL-C were calculated by HLD-C subtracted from total cholesterol. Further, EDTA anticoagulated whole blood was collected from patients for HBA1c tests and individual genomic DNA extraction. Lp(a) concentrations were measured in serum using an immunoturbidimetric method. Interassay variation for samples in the 
centralized laboratory was $<7 \%$. The reference material is SRM2B IFCC/WHO for the two $\operatorname{Lp}(\mathrm{a})$ assay reagents. All results were measured on the condition of quality control being normal.

KIV-2 repeat copy number was determined according to the repeat copy number, using a Taqman probe-based quantitative PCR technique, as reported in the literature [21]. The basic detection steps are as follows: Specific probes for KIV-2 and endogenous-single-copy control housekeeping gene were synthesized. Applied Biosystems $^{\circ} 9600$ Real-Time PCR Systems was used with a total reaction system of $20 \mu \mathrm{l}$, which included premix Ex Taq $(2 \times) 10 \mu l$, specific primer $0.4 \mu \mathrm{l}$, Taqman probe 0 . $8 \mu \mathrm{l}$, and DNA template $2 \mu \mathrm{l}$ (concentration 3-5 ng/ $\mu \mathrm{l}$ ).

\section{Statistical analysis}

Statistical analysis was conducted using SPSS 18.0 software. Data with Gaussian distribution were expressed as the mean \pm standard deviation (SD), and data with nonGaussian distribution were expressed as the median (interquartile range). For further statistical analysis, data with non-Gaussian distribution log transformed into Gaussian distribution. An independent sample t-test or one-way analysis of variance (ANOVA) was used to compare the mean of each group; the $\chi^{2}$ test in nonparametric statistics was used to compare categorical variables among groups. Logistic regression (backwardstepwise method) analysis was used to estimate CAHD risk for various indicators. This study considered $P<0$. 05 as the significance criterion.

\section{Results}

\section{Clinical data and characteristics of study subjects}

A total of 884 subjects were enrolled in this study, including 484 CAHD patients and 400 non-CAHD individuals. Table 1 shows clinical characteristics and risk factors of all subjects, including gender, age, blood-lipid indicators, hs-CRP, Lp(a) mass concentration and particle concentration as well as KIV-2 copy number. For CAHD and non-CAHD groups, an independentsample t-test was used to compare the mean of various indicators with Gaussian distribution, and the $\chi^{2}$ test was used to compare the difference in rates of categorical variables. The means of Apo(B), Lp(a) mass concentration and particle concentration, KIV-2 copy number, HDL-C, age, HBA1c and hs-CRP of patients in the CAHD group were all significantly higher or lower than the non-CAHD group $(P<0.05)$. Differences between the means of the two groups were not significant for other indicators, such as total cholesterol, LDL-C, non-HDL-C and triglycerides. Indicators with differences were believed to be possible risk factors for CAHD. Both average $\mathrm{Lp}$ (a) mass concentration and particle concentration of patients in the CAHD group were higher than in the non-CAHD group, while the KIV-2 copy number of patients in the CAHD group was lower than the nonCAHD group. This result fully confirmed that high Lp(a) level and low KIV-2 copy number were closely related to the occurrence of CAHD and were possibly independent risk factors for CAHD.

\section{Comparison of various clinical indicators among subgroups with different plaque types}

ANOVA and $\chi^{2}$ test results for soft-plaque, calcifiedplaque and mixed-plaque subgroups were as follows: There were significant differences in the means of respective sub-groups for age, gender, total cholesterol, LDL-C, non-HDL-C, and Lp(a) mass concentration and particle concentration. The results are shown in Table 2 . Further intergroup pair-wise comparisons are as follows: Lp(a) levels expressed as mass concentration and particle concentration in the soft-plaque subgroup were higher than the other two groups. Total cholesterol in the soft-plaque subgroup was higher than the mixedplaque subgroup. Non-HDL-C in the soft-plaque subgroup was higher than in the calcified- plaque subgroup and between-group differences were not significant for the remaining indicators.

\section{Linear regression results of $L p(a)$ mass concentration and particle concentration}

For correlation analysis of all subjects and to draw a scatter plot, $\mathrm{Lp}(\mathrm{a})$ particle concentration was considered the abscissa and $\mathrm{Lp}(\mathrm{a})$ mass concentration was considered the ordinate for linear regression analysis, and results are indicated in Fig. 1a. The results of two different concentrations showed a good linear relationship, and the linear regression equation of particle concentration to mass concentration could be obtained: $\quad \mathrm{Y}_{\mathrm{Lp}(\mathrm{a}) \text {-mass }}=6.565 \mathrm{X}_{\mathrm{Lp}(\mathrm{a}) \text {-particle, }}$ and the correlation coefficient $\mathrm{R}^{2}$ was $0.852(P<0.001)$. At present, abnormal $\mathrm{Lp}(\mathrm{a})$ levels in clinical practice are mainly evaluated based on a reagent instruction for use. For $\mathrm{Lp}(\mathrm{a})$ mass concentration, $300 \mathrm{mg} / \mathrm{L}$ is usually taken as a cutoff value, i.e., an individual's test result $\leq 300 \mathrm{mg} / \mathrm{L}$ is considered normal and $>300 \mathrm{mg} / \mathrm{L}$ is considered elevated. For particle concentration, $75 \mathrm{nmol} / \mathrm{L}$ is the cutoff value, i.e., $\leq 75 \mathrm{nmol} / \mathrm{L}$ is normal, and $>75 \mathrm{nmol} / \mathrm{L}$ is considered elevated. According to the criteria, all subjects were gauged as normal or abnormal in $\mathrm{Lp}$ (a) mass concentration and particle concentration, respectively. The number and proportion of normal and abnormal cases were respectively counted. Kappa consistency test was then used to compare the consistency between the two kinds of $\mathrm{Lp}(\mathrm{a})$ results. The number of subjects with elevated $\mathrm{Lp}(\mathrm{a})$ mass concentration was significantly higher than that of subjects with elevated particle 
Table 1 Baseline clinical characteristics and risk factors in patients with CAHD and without

\begin{tabular}{|c|c|c|c|c|}
\hline & $\begin{array}{l}\text { CAHD group } \\
n=484(54.8 \%)\end{array}$ & $\begin{array}{l}\text { Non-CAHD group } \\
n=400(45.2 \%)\end{array}$ & $t / x^{2}$ & $P$ value \\
\hline Age(y) & $62.46 \pm 9.06$ & $56.39 \pm 9.57$ & $-9.405^{c}$ & 0.000 \\
\hline Sex (male), n (\%) & $277(57.2 \%)$ & $170(42.5 \%)$ & $19.635^{b}$ & 0.000 \\
\hline Diabetes mellitus,n(\%) & $108(22.3 \%)$ & $28(7.0 \%)$ & $39.451^{\mathrm{b}}$ & 0.000 \\
\hline Total cholesterol (mmol/L) & $4.79 \pm 1.08$ & $4.84 \pm 0.98$ & 0.710 & 0.478 \\
\hline LDL-C (mmol/L) & $3.15 \pm 0.99$ & $3.15 \pm 0.89$ & 0.016 & 0.988 \\
\hline $\mathrm{HDL}-\mathrm{C}(\mathrm{mmol} / \mathrm{L})$ & $1.33 \pm 0.59$ & $1.42 \pm 0.41$ & $2.661^{c}$ & 0.008 \\
\hline non-HDL-C (mmol/L) & $3.46 \pm 1.17$ & $3.42 \pm 0.94$ & -0.593 & 0.553 \\
\hline Triglycerides (mmol/L) & $1.99 \pm 1.27$ & $1.83 \pm 1.27$ & -1.801 & 0.072 \\
\hline Apo(B) $(g / L)$ & $1.09 \pm 0.35$ & $1.05 \pm 0.31$ & -1.847 & 0.065 \\
\hline Lp(a)-mass (mg/L) ${ }^{a}$ & $181(69-346)$ & $130(66-287)$ & $-3.121^{c}$ & 0.002 \\
\hline Lp(a)-particle $(\mathrm{nmol} / \mathrm{L})^{\mathrm{a}}$ & $19.1(8.8-44.9)$ & $14.4(7.6-32.3)$ & $-2.543^{c}$ & 0.011 \\
\hline KIV-2 copy numbers (copies) & $14.11 \pm 6.13$ & $15.14 \pm 7.11$ & $2.205^{c}$ & 0.040 \\
\hline $\mathrm{HbA1C}(\%)$ & $6.12 \pm 1.02$ & $5.83 \pm 0.66$ & $-4.824^{c}$ & 0.000 \\
\hline $\mathrm{hs}-\mathrm{CRP}(\mathrm{mg} / \mathrm{L})^{\mathrm{a}}$ & $0.91(0.44-2.02)$ & $0.68(0.38-1.62)$ & $-2.967^{c}$ & 0.003 \\
\hline
\end{tabular}

Continuous variables are expressed as mean \pm SD or ${ }^{a}$ median (interquartile range) and compared using ' $t$ ' test

${ }^{b}$ Categorical variables are expressed a numbers (\%) and compared using the chi-square test

${ }^{c}$ Compared with control group, $\mathrm{P}<0.05$

concentration (231 vs. 108 in 884 subjects), and the calculated kappa value from the consistency test was 0.536 $(P<0.01)$. Considering kappa value $>0.75$ the acceptable criterion for consistency, judgment of the same patient on the basis of two $\operatorname{Lp}(\mathrm{a})$ concentrations was obviously inconsistent. Therefore, varying judgments on the basis of two concentrations bring complexity to the clinician's decision in assessing risk and evaluating the effect of treatment in CAHD patients.
The relationship between $\mathrm{Lp}(\mathrm{a})$ concentration and KIV-2 copy number distribution in the Chinese Han population Considering the LPA gene KIV-2 copy number the abscissa and $\mathrm{LP}(\mathrm{a})$ particle concentration and mass concentration the ordinates, an XY scatter plot for all subjects was drawn, as shown in Fig. 1b. Pearson correlation analysis showed that the coefficient $r$ between particle concentration or mass concentration and KIV-2 copy number was -0.145 or -0.135 , respectively. Both variables had $P<0.01$,

Table 2 Relationship between risk factors and different nature plaques in CAHD patients compared using ANOVA ( $n=484$ )

\begin{tabular}{|c|c|c|c|c|c|c|c|}
\hline & $\begin{array}{l}\text { Soft plaque } \\
n=259\end{array}$ & $\begin{array}{l}\text { Calcified plaque } \\
n=129\end{array}$ & $\begin{array}{l}\text { Mixed plaque } \\
n=96\end{array}$ & $P$ value & $P_{1}$ value & $P_{2}$ value & $P_{3}$ value \\
\hline Age (years) & $61.37 \pm 9.13$ & $63.41 \pm 8.72$ & $64.38 \pm 8.88$ & 0.010 & 0.045 & 0.006 & 0.446 \\
\hline Sex (male), n (\%) & $145(56 \%)$ & $64(49.6 \%)$ & $68(70.8 \%)$ & $0.020^{b}$ & / & / & / \\
\hline Total cholesterol (mmol/L) & $4.90 \pm 1.11$ & $4.68 \pm 1.01$ & $4.62 \pm 1.04$ & 0.045 & 0.080 & 0.028 & 0.640 \\
\hline LDL-cholesterol (mmol/L) & $3.27 \pm 1.01$ & $3.08 \pm 0.88$ & $2.93 \pm 0.99$ & 0.009 & 0.080 & 0.003 & 0.266 \\
\hline HDL-cholesterol (mmol/L) & $1.32 \pm 0.38$ & $1.42 \pm 0.67$ & $1.24 \pm 0.43$ & 0.076 & 0.110 & 0.275 & 0.026 \\
\hline Non-HDL cholesterol (mmol/L) & $3.58 \pm 1.11$ & $3.27 \pm 1.36$ & $3.37 \pm 1.05$ & 0.041 & 0.017 & 0.139 & 0.498 \\
\hline Triglycerides (mmol/L) & $2.00 \pm 1.26$ & $1.80 \pm 1.04$ & $2.20 \pm 1.52$ & 0.076 & 0.168 & 0.181 & 0.023 \\
\hline Apo(B) $(g / L)$ & $1.12 \pm 0.34$ & $1.06 \pm 0.38$ & $1.06 \pm 0.31$ & 0.178 & 0.128 & 0.144 & 0.983 \\
\hline Lp(a)-mass, (mg/L) & 200(69-383) & 166(77-293) & 149(64-279) & 0.025 & 0.049 & 0.018 & 0.662 \\
\hline Lp(a)-particle, $(\mathrm{nmol} / \mathrm{L})^{\mathrm{a}}$ & $21.5(5.3-52)$ & $18.6(5.3-43.1)$ & $17.1(7.6-28.01)$ & 0.006 & 0.038 & 0.003 & 0.391 \\
\hline KIV-2 copy numbers (copies) & $14.31 \pm 6.94$ & $13.84 \pm 5.25$ & $13.87 \pm 4.20$ & 0.768 & 0.543 & 0.587 & 0.979 \\
\hline $\mathrm{HbA1C}(\%)$ & $6.10 \pm 1.08$ & $6.12 \pm 0.89$ & $6.18 \pm 0.99$ & 0.783 & 0.848 & 0.485 & 0.657 \\
\hline HS-CRP $(\mathrm{mg} / \mathrm{L})^{a}$ & $0.91(0.45-1.79)$ & $0.86(0.43-1.88)$ & $0.92(0.45-2.52)$ & 0.515 & 0.292 & 0.456 & 0.814 \\
\hline
\end{tabular}

Notice: $P$ value calculated among three groups; $P_{1}$ value compared between soft and calcified plaque; $P_{2}$ value compared between soft and mixed plaque; $P_{3}$ value compared between calcified and mixed plaque

${ }^{\text {a }}$ Results shown as mean \pm SD or median (interquartile range)

${ }^{\mathrm{b}}$ Results compared using the chi-square test 

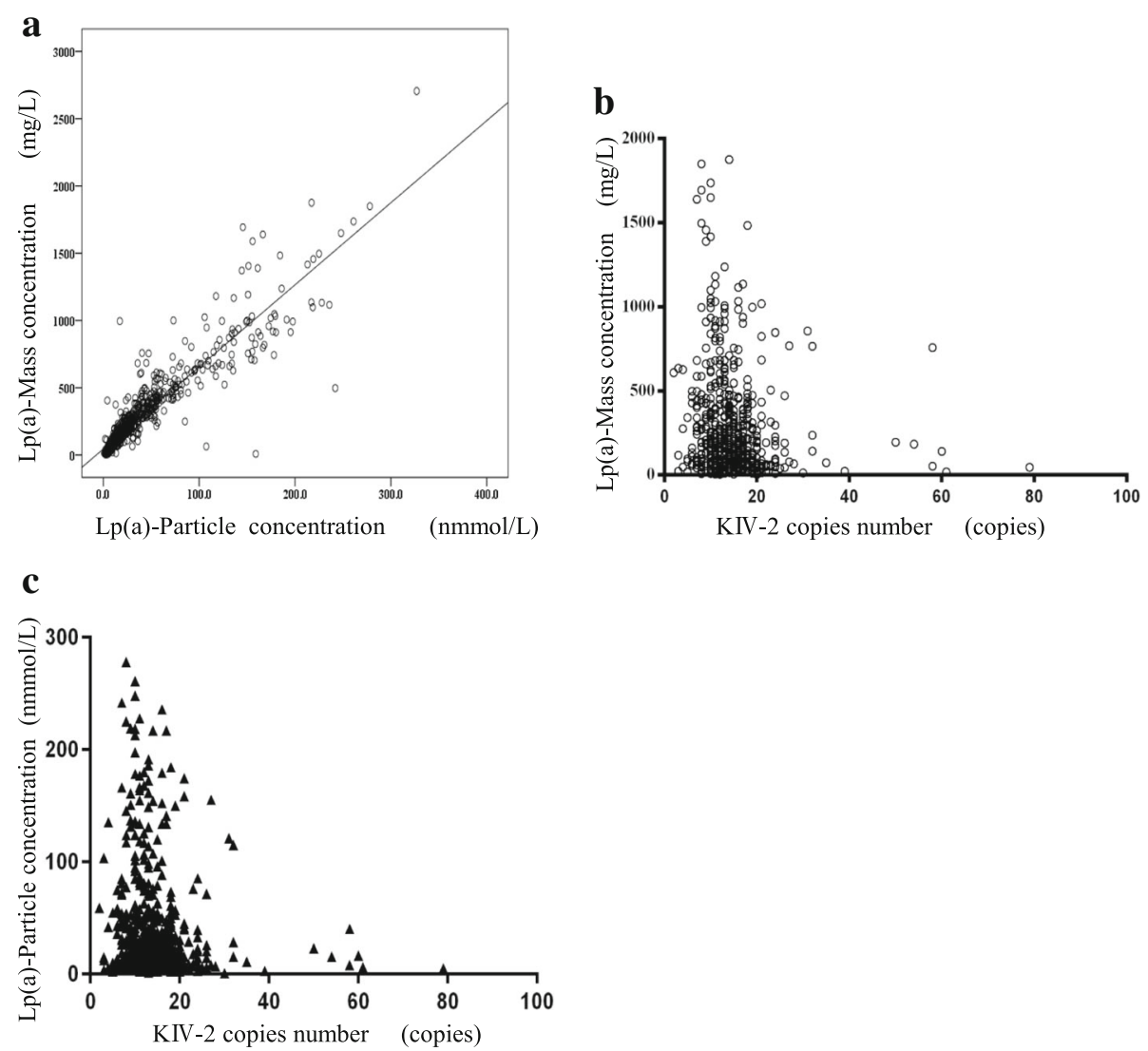

Fig. 1 a Shows an XY scatter plot of $L p(a)$ mass and particle concentration, and the linear relationship with a regression equation: $Y_{L p(a)-m a s s}=6.565 X_{L p(a)-p a r t i c l e r}$ $R^{2}=0.852$, $P<0.001$. b Shows an XY scatter plot of KIV-2 copy number and Lp(a) mass concentration for all participants. The abscissa is KIV-2 copy number, and the left ordinate is $L p(a)$ mass concentration. c Shows KIV-2 copy number and Lp(a) particle concentration. The abscissa is KIV-2 copy number, and the left ordinate (right axis) is $L p(a)$ particle concentration. There is a negative correlation between $L p(a)$ level and KIV-2 copy number, i.e., the smaller the KIV-2 copy number the higher the Lp(a) concentration

indicating that the $\mathrm{Lp}(\mathrm{a})$ level was negatively related to the KIV-2 copy number: The larger the KIV-2 copy number was, the lower the $\operatorname{Lp}(\mathrm{a})$ concentration. This conclusion was accurate for the $\mathrm{Lp}(\mathrm{a})$ levels described based on both particle concentration and mass concentration. Numerically, particle concentration was more closely related to KIV-2 copy number than mass concentration.

Distribution histograms of $\mathrm{Lp}(\mathrm{a})$ mass concentration and Lp(a) particle concentration as well as KIV-2 copy number were drawn via software in all study subjects, as shown in Fig. 2. For all subjects, median $\mathrm{Lp}(\mathrm{a})$ mass concentration was $146 \mathrm{mg} / \mathrm{L}$ (interquartile range: 67-342 $\mathrm{mg} / \mathrm{L}$ ), median $\mathrm{Lp}$ (a) particle concentration was $17 \mathrm{nmol} / \mathrm{L}$ (interquartile range: $8-41 \mathrm{nmol} / \mathrm{L}$ ), and median $\mathrm{KIV}-2$ copy number was 14 (interquartile range: 10-17 copies), respectively.

\section{Differences in average $\operatorname{Lp}(\mathrm{a})$ concentration and CAHD patient proportion among groups with different KIV-2 copy numbers}

All subjects were divided into four groups: Groups Q1-Q4 were based on interquartile range of the KIV-
2 copy number (measured in the laboratory); thus, the 25th, 50th and 75th percentiles were 10, 14, and 17 copies, respectively. ANOVA results indicated that the among-group mean $\mathrm{Lp}(\mathrm{a})$ was significantly different, except between the last two groups, and the mean in group Q1 was the highest. In the comparison of CAHD prevalence among different groups, the $x^{2}$ test results showed there was no significant difference in CAHD percent among the groups (Pearson Chi-square value was 5.710, $P=0.127$ ). All results are shown in Fig. 3.

Risk estimation of various clinical conventional risk factor indicators and Lp(a) and KIV-2 copy number on CAHD occurrence

For all subjects, dependent or independent variables were explored for CAHD, considering all CAHD risk factors, such as gender, age, KIV-2 copy number, HDL-C, Lp(a) mass concentration/particle concentration, conventional lipid indicators, and hsCRP and HBA1c as independent variables. The backward-stepwise method was used for 


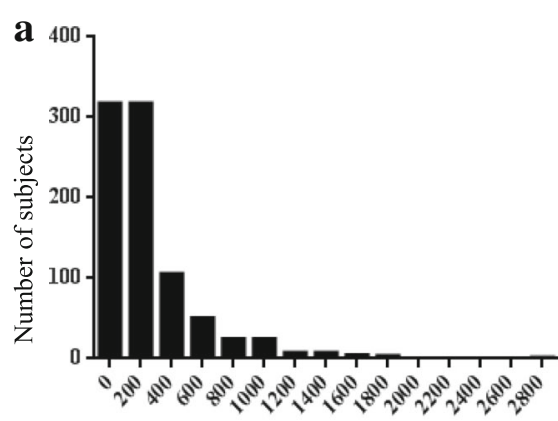

Lp(a)-mass concentration (mg/L)

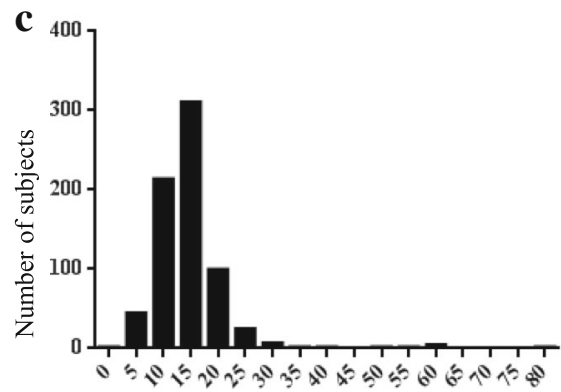

KIV-2 copies number (copies)

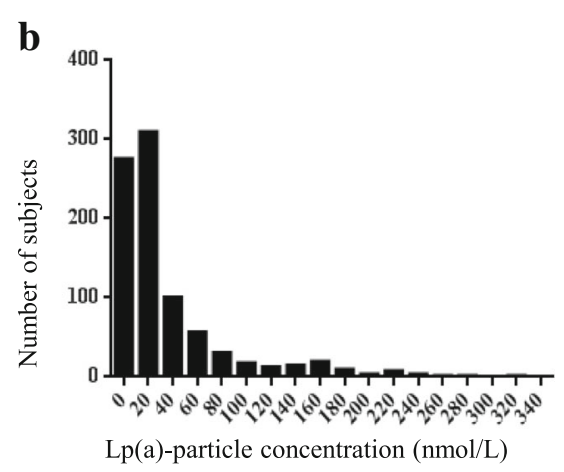

Fig. 2 Represents Lp(a) mass concentration and particle concentration, and KIV-2 population distributions in the Chinese Han ethnic population; a $L p(a)$ mass concentration distribution histogram; b Lp(a) particle concentration distribution histogram; c KIV-2 copy number distribution histogram

logistic regression analysis to calculate respective odds ratios (OR). In the final regression equation, 6 indicators, KIV-2 copy number, Lp(a), HBA1c, age, gender and HDL-C, were retained. High Lp(a) levels (both mass concentration and particle concentration), high HBA1c and increasing age were independent risk factors for CAHD. In contrast, high HDL-C, large KIV-2 copy number and female gender were protective factors for CAHD. A tree diagram of the statistical results is shown in Fig. 4. The OR value calculated by using particle concentration was greater than the OR value calculated by using mass concentration, indicating that Lp(a) levels expressed by particle concentration were more closely related to CAHD than mass concentration.

\section{Discussion}

Since its discovery, elevated $\mathrm{Lp}(\mathrm{a})$ seems to be related to CAHD but remains controversial, as $\mathrm{Lp}(\mathrm{a})$ is an independent risk factor for CAHD due to its structural peculiarity and complexity [22, 23]. Some studies even consider that $\operatorname{Lp}(\mathrm{a})$ is related to the severity of CAHD $[4,24,25]$. This study indicates that in addition to age, gender, HBA1c, hs-CRP and other conventional risk factors, the difference in $\mathrm{Lp}$ (a) levels between CAHD and non-CAHD groups was significant, as both particle concentration and mass concentration in the CAHD group were significantly higher than in the non-CAHD group. This result indicates that in the Chinese Han population, elevated $\mathrm{Lp}(\mathrm{a})$ is also an independent risk factor for CAHD and is consistent with the conclusion of a crosssectional study on the Chinese Han population by Cai et al. [26]. For comparison, the results obtained from various subgroups with different plaque types indicated significant differences in age, gender, cholesterol, LDL-C, non-HDL-C and Lp(a) levels in the soft-plaque subgroup, especially $\mathrm{Lp}(\mathrm{a})$ particle concentration, which had particular value in predicting soft plaque. This outcome was a new discovery in the clinical application of $\mathrm{Lp}(\mathrm{a})$. Compared to coronary angiography, the CTA examination used in this study could determine the type of atherosclerotic plaque and assess its stability in addition to being convenient and causing very little damage. An unstable plaque is prone to rupture, thus promoting thrombus formation and causing a serious cardiovascular event. Hence, identifying coronary arterial soft plaque helps identify unstable plaque. Hopefully, the correlation between $\operatorname{Lp}(\mathrm{a})$ levels and soft plaque makes $\mathrm{Lp}(\mathrm{a})$ an auxiliary indicator for gauging the stability of atherosclerotic plaque.

This study found good linear relationship between two Lp(a) concentrations by comparing the two and deduced that the conversion equation was: mass concentration = particle concentration $\times 6.565$. However, the recommended conversion factor in the reagent instruction for use was 4.167 , which was significantly different from the 

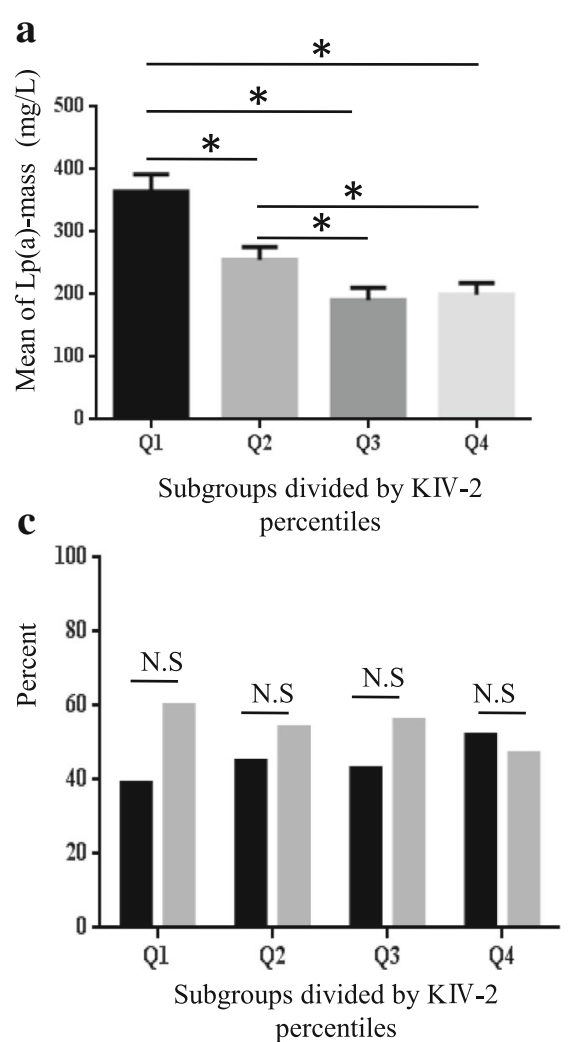

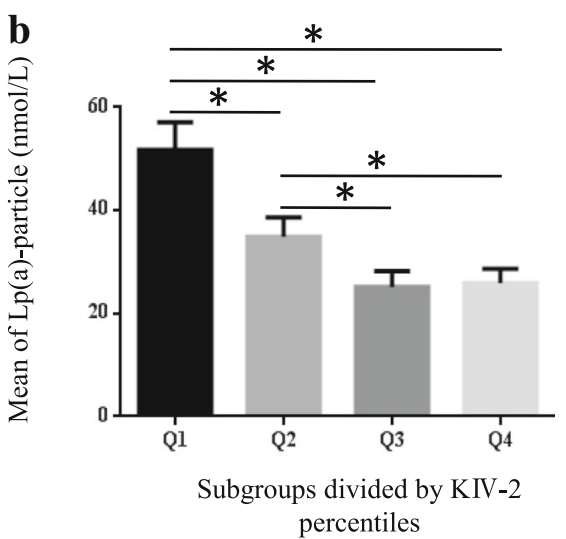

non-CAHD CAHD

Fig. 3 Comparison of Lp(a) mass concentration, particle concentration, and percent of CAHD among KIV-2 subgroups; * P > 0.05; N.S: no significance; a Differences of Lp(a) mass concentration in CAHD and non-CAHD participants among different KIV-2 subgroups; b Differences of Lp(a) particle concentration in CAHD, non-CAHD participants among different KIV-2 subgroups; $\mathbf{c}$ Differences of proportion in CAHD, non-CAHD participants among different KIV-2 subgroups

estimated conversion factor. Additionally, while using two concentrations of $\mathrm{Lp}(\mathrm{a})$ to gauge the same individual based on their respective reference intervals, i.e., mass concentration $\leq 300 \mathrm{mg} / \mathrm{L}$ and particle concentration $\leq 75 \mathrm{nmol} / \mathrm{L}$, the conclusions were not exactly the same. The proportion of patients with elevated mass concentration was significantly higher than the proportion of patients with elevated particle concentration, indicating that mass concentration may overestimate the $\operatorname{Lp}(\mathrm{a})$ level of the patient, or that the biological reference interval with particle concentration $\leq 75 \mathrm{nmol} / \mathrm{L}$ is not suitable for the Chinese Han population. Therefore, interchangeability between particle concentration and mass concentration needs to be studied further in a large sample size to establish a biological reference interval suitable for the Chinese Han population. Currently, direct conversion is not suitable, and particle concentration should be used to report results as far as possible. Some studies even propose that total mass concentration of $\mathrm{Lp}(\mathrm{a})$, as an indicator, should not be advocated [27].

The present study indicates that the KIV-2 copy number variation of the LPA gene is the key factor determining
$\mathrm{Lp}$ (a) concentration, and its copy number varies from 2 to $>40$ in the Chinese population. Some studies have indicated that there is racial heterogeneity in LPA gene SNP loci $[28,29]$. Therefore, it is hypothesized that there may be racial differences in the KIV-2 copy number. In this study, Lp(a) levels and KIV-2 copy number distributions in the Chinese population were observed. The results showed that the median $\mathrm{Lp}(\mathrm{a})$ mass concentration was $146 \mathrm{mg} / \mathrm{L}$ and that of particle concentration was $17 \mathrm{nmol} / \mathrm{L}$ in the Chinese population, which was significantly lower than those in other racial populations from different countries, prompting that $\mathrm{Lp}(\mathrm{a})$ distribution in the Chinese population has particularity. The Lp(a) biological reference interval is established based on the 75th percentile of Caucasians. KIV-2 copy number distribution in the Chinese Han population with an average copy number of 14 is also different from other races [30]. As KIV contains 9 other single-copy repeats, in addition to KIV-2, the KIV sequence actually contains 23 repeats. The present study also confirmed the negative relationship between KIV-2 copy number and $\mathrm{Lp}$ (a) concentration. Linear regression analysis showed that the larger the KIV-2 copy number, the lower the Lp(a) concentration in an individual's blood, which is 


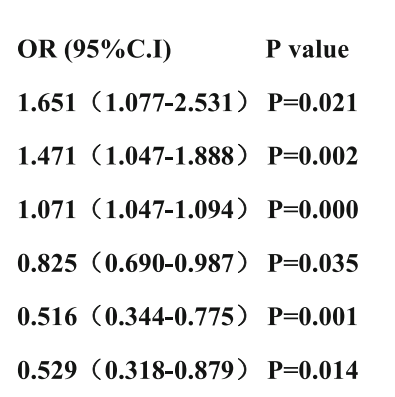

$\mathbf{a}$

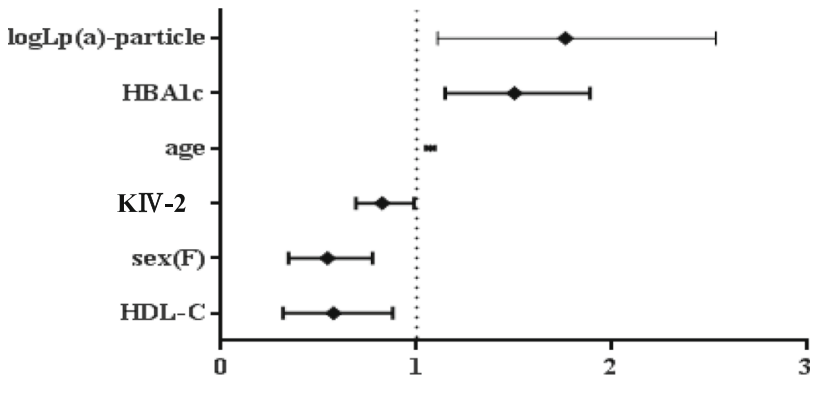

b

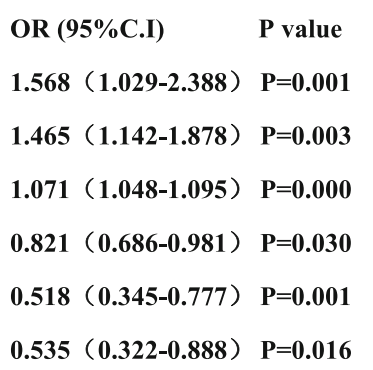

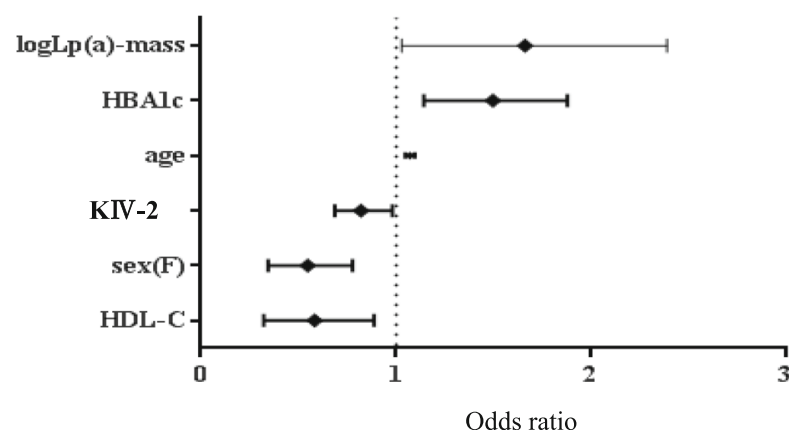

Fig. 4 Tree diagrams of logistic regression analysis on various possible risk factors for CAHD; a Analysis results of Lp(a)-particle and other indicators; $\mathbf{b}$ Analysis results of Lp(a)-mass and other indicators

consistent with Paultre et al.'s study conclusion that apo (a) subtype with fewer KIV copy numbers is related to higher $\mathrm{Lp}$ (a) concentration and is a risk factor, thus predicting CAHD [31]. The population was then divided into four groups based on KIV-2 copy number and quartile: The results showed that mean $\mathrm{Lp}(\mathrm{a})$ concentration in groups Q1 and Q2 with fewer copy numbers was higher than that in groups Q3 and Q4. Additionally, this number was higher in group Q1 than in group Q2, and there was no significant difference between groups Q3 and Q4, indicating continuous change between KIV-2 copy number and Lp(a) concentration, with a cumulative effect.

Considering the CAHD risk factors as dependent or independent variables, logistic regression was performed, and the results were as follows. Besides high HBA1c,advanced age, low HDL-C level, male gender and other factors, such as high $\mathrm{Lp}(\mathrm{a})$ level and low KIV-2 copy number, were also risk factors for CAHD occurrence. Elevated $\mathrm{Lp}$ (a) level had the highest OR value for CAHD and large KIV-2 copy number could lower the risk. Additionally, analysis of CAHD patients in groups with different KIV-2 copy numbers revealed no significant difference among the different groups in this study, indicating that the KIV-2 copy number had no significant effect on CAHD. This finding was inconsistent with the logistic regression results, which indicated the relationship between the KIV-2 copy number at the gene level and CAHD. Effects of the KIV-2 copy number at the gene level, however, are limited. KIV-2 copy number variation also needs other factors such as epigenetic, protein expression, assembly and environment to exert effects collectively. Additionally, there are also reports about uncertain causality between LPA gene mutation and CAHD [8, 32].

Since this study included cases and controls, biases may have been introduced. The accuracy of KIV-2 copy number detection was also one of the factors affecting the analysis of results. This study used the qPCR technique developed by Lanktree et al. [21, 33], however, it measured the average KIV-2 copy number of an individual diploid. Since two haploids might contain different KIV-2 copy numbers, this method cannot effectively differentiate between the effects of individual haploids [34]; for example, the 16/28 genotype had the same PCR measurement result as the $22 / 22$ genotype, but gene expression or patients' phenotype might be different between the two gene types.

\section{Conclusions}

In summary, the present study explored the relationship between " $\mathrm{L}(\mathrm{a})$ particle concentration and mass concentration distribution" and "CAHD" in the "Chinese Han population", confirming that high $\mathrm{Lp}(\mathrm{a})$ concentration is a risk factor for CAHD and that $\mathrm{Lp}(\mathrm{a})$ particle 
concentration has auxiliary diagnostic value to gauge the type of coronary arterial plaque. A qPCR technique was used to detect the KIV-2 copy number of subjects and its relationship with $\mathrm{Lp}(\mathrm{a})$ concentration, and its population distribution was then assessed as well as its role in risk assessment of CAHD.

\section{Abbreviations}

CAHD: Coronary atherosclerotic heart disease; CNV: Copy number variation; CTA: Computerized tomography angiography; HDL-C: High density lipoprotein-cholesterol; hs-CRP: Hypersensitive $\mathrm{C}$ reactive protein; HU: Hounsfield unit; KIV-2: Kringle IV type 2; LDL-C: Low density lipoproteincholesterol; Lp(a): Lipoprotein(a); SNP: Single nucleotide polymorphism

\section{Acknowledgements}

We thank the patients for their contribution to this study and thank all the members of our laboratory for stimulating discussion.

\section{Funding}

This project was supported by the Natural Science Foundation of China (grant nos. 81671599 and 81372212), Shanghai Municipal Health and Planning Commission (No. 20154Y0115), Science and Technology Committee of Shanghai (No. 17441902400, 17JC1401002), Pudong New Area Science and Technology Commission (No. PKJ2015-Y18).

\section{Availability of data and materials}

The datasets used and/or analyzed during the current study are available from the corresponding author on reasonable request.

\section{Authors' contributions}

LS and LF participated in the study design. MZ, CC, LX were involved in the conduct of the study and data collection. FW and MY made contributions to data analysis and results interpretation. LS, MZ and LF wrote and modified the manuscript and prepared tables and figures. All authors read and approved the final manuscript.

\section{Ethics approval and consent to participate}

The project was approved by the Ethical Committee of Shanghai East hospital (2015-LS-011). All subjects signed informed consent forms.

\section{Competing interests}

The authors declare that they have no competing interests.

\section{Publisher's Note}

Springer Nature remains neutral with regard to jurisdictional claims in published maps and institutional affiliations.

\section{Received: 10 February 2018 Accepted: 23 April 2018}

Published online: 10 May 2018

\section{References}

1. Nordestgaard BG, Chapman MJ, Ray K, Boren J, Andreotti F, Watts GF, Ginsberg H, Amarenco P, Catapano A, Descamps OS, et al. Lipoprotein(a) as a cardiovascular risk factor: current status. Eur Heart J. 2010;31:2844-53. https://doi.org/10.1093/eurhearti/ehq386.

2. Dube JB, Boffa MB, Hegele RA, Koschinsky ML. Lipoprotein(a): more interesting than ever after 50 years. Curr Opin Lipidol. 2012;23:133-40. https://doi.org/10.1097/MOL.0b013e32835111d8.

3. Kamstrup PR, Tybjærg-Hansen A, Nordestgaard BG. Extreme lipoprotein(a) levels and improved cardiovascular risk prediction. J Am Coll Cardiol. 2013; 61:1146-56. doi:http://dx.doi.org/10.1016/j.jacc.2012.12.023

4. O'Donoghue ML, Morrow DA, Tsimikas S, Sloan S, Ren AF, Hoffman EB, Desai NR, Solomon SD, Domanski M, Arai K, et al. Lipoprotein(a) for risk assessment in patients with established coronary artery disease. J Am Coll Cardiol. 2014;63:520-7. doi:http://dx.doi.org/10.1016/j.jacc.2013.09.042

5. Bucci M, Tana C, Giamberardino MA, Cipollone F. Lp(a) and cardiovascular risk: investigating the hidden side of the moon. Nutr Metab Cardiovasc Dis. 2016;26:980-6. https://doi.org/10.1016/j.numecd.2016.07.004.
6. Forbes CA, Quek RG, Deshpande S, Worthy G, Wolff R, Stirk L, Kleijnen J, Gandra SR, Djedjos S, Wong ND. The relationship between Lp(a) and CVD outcomes: a systematic review. Lipids Health Dis. 2016;15:95. doi:10.1080/ 10408363.2016.1188055. 10.1186/s12944-016-0258-8

7. Noureen A, Fresser F, Utermann G, Schmidt K. Sequence variation within the KIV-2 copy number polymorphism of the human LPA gene in African, Asian, and European populations. PLoS One. 2015;10:e0121582. https://doi. org/10.1371/journal.pone.0121582.

8. Danik JS, Buring JE, Chasman DI, Zee RY, Ridker PM, Glynn RJ. Lipoprotein(a) polymorphisms in the LPA gene, and incident venous thromboembolism among 21483 women. J Thromb Haemost. 2013;11:205-8. doi:10.3389/ fimmu.2012.00367. doi:10.1111/jth.12056

9. Bjornsson E, Gudbjartsson DF, Helgadottir A, Gudnason T, Gudbjartsson T, Eyjolfsson K, Patel RS, Ghasemzadeh N, Thorleifsson G, Quyyumi AA, et al. Common sequence variants associated with coronary artery disease correlate with the extent of coronary atherosclerosis. Arterioscler Thromb Vasc Biol. 2015;35:1526-31. https://doi.org/10.1161/atvbaha.114.304985.

10. Lee SR, Prasad A, Choi YS, Xing C, Clopton P, Witztum JL, Tsimikas S. The LPA gene, ethnicity, and cardiovascular events. Circulation. 2016; https://doi. org/10.1161/circulationaha.116.024611.

11. Burgess S, Harshfield E. Mendelian randomization to assess causal effects of blood lipids on coronary heart disease: lessons from the past and applications to the future. Curr Opin Endocrinol Diabetes Obes. 2016;23: 124-30. https://doi.org/10.1097/med.0000000000000230.

12. Jansen $\mathrm{H}$, Samani $\mathrm{NJ}$, Schunkert H. Mendelian randomization studies in coronary artery disease. Eur Heart J. 2014;35:1917-24. https://doi.org/10. 1093/eurheartj/ehu208.

13. Lippi G, Franchini M, Targher G. Screening and therapeutic management of lipoprotein(a) excess: review of the epidemiological evidence, guidelines and recommendations. Clin Chim Acta. 2011;412:797-801. doi:http://dx.doi. org/10.1016/j.cca.2011.01.018

14. Stein EA, Raal F. Future directions to establish lipoprotein(a) as a treatment for atherosclerotic cardiovascular disease. Cardiovasc Drugs Ther. 2016;30: 101-8. https://doi.org/10.1007/s10557-016-6654-5.

15. Afshar M, Kamstrup PR, Williams K, Sniderman AD, Nordestgaard BG, Thanassoulis G. Estimating the population impact of Lp(a) lowering on the incidence of myocardial infarction and aortic stenosis-brief report. Arterioscler Thromb Vasc Biol. 2016;36:2421-3. https://doi.org/10.1161/ atvbaha.116.308271.

16. Hoover-Plow J, Huang M. Lipoprotein(a) metabolism: potential sites for therapeutic targets. Metabolism. 2013;62:479-91. https://doi.org/10.1016/j. metabol.2012.07.024

17. Kajinami K. Lipoprotein(a): revisiting a next therapeutic target. Circ J. 2016; 80:329-31. https://doi.org/10.1253/circj.CJ-15-1381.

18. Konerman M, Kulkarni K, Toth PP, Jones SR. Lipoprotein(a) particle concentration and lipoprotein(a) cholesterol assays yield discordant classification of patients into four physiologically discrete groups. J Clin Lipidol. 2012;6:368-73. doi:http://dx.doi.org/10.1016/j.jacl.2012.01.004

19. Zhu L, Lu Z, Zhu L, Ouyang X, Yang Y, Feng Y, Li P, Ye W, Zhang X, Zhang $X$, Song Y. Plasma lipoprotein(a) levels are associated with the severity of coronaryheart disease in Han Chinese people. Turk J Med Sci. 2016;46:103341. https://doi.org/10.3906/sag-1502-74.

20. Wu Z, Sheng H, Chen Y, Tang J, Liu Y, Chen Q, Lu L, Jin W. Copy number variation of the lipoprotein(a) (LPA) gene is associated with coronary artery disease in a southern Han Chinese population. Int J Clin Exp Med. 2014;7:3669-77.

21. Kamstrup PR, Tybjaerg-Hansen A, Steffensen R, Nordestgaard BG. Genetically elevated lipoprotein(a) and increased risk of myocardial infarction. JAMA. 2009;301:2331-9. https://doi.org/10.1001/jama.2009.801.

22. Maranhao RC, Carvalho PO, Strunz CC, Pileggi F. Lipoprotein (a): structure, pathophysiology and clinical implications. Arq Bras Cardiol. 2014;103:76-84.

23. Kunutsor SK, Khan H, Nyyssonen K, Laukkanen JA. Is lipoprotein (a) protective of dementia? Eur J Epidemiol. 2016;31:1149-52. https://doi.org/ 10.1007/s10654-016-0184-0.

24. Sposito AC, Mansur AP, Maranhao RC, Martinez TR, Aldrighi JM, Ramires JA. Triglyceride and lipoprotein (a) are markers of coronary artery disease severity among postmenopausal women. Maturitas. 2001;39:203-8.

25. Chen J, Zhang Y, Liu J, Chen MH, Guo YL, Zhu CG, Xu RX, Dong Q, Li JJ. Role of lipoprotein(a) in predicting the severity of new on-set coronary artery disease in type 2 diabetics: a Gensini score evaluation. Diab Vasc Dis Res. 2015;12:258-64. https://doi.org/10.1177/1479164115579004. 
26. Cai DP, He YM, Yang XJ, Zhao X, Xu HF. Lipoprotein (a) is a risk factor for coronary artery disease in Chinese Han ethnic population modified by some traditional risk factors: a cross-sectional study of 3462 cases and 6125 controls. Clin Chim Acta. 2015;451:278-86. https://doi.org/10.1016/..cca.2015. 10.009 .

27. Enkhmaa B, Anuurad E, Berglund L. Lipoprotein (a): impact by ethnicity and environmental and medical conditions. J Lipid Res. 2016;57:1111-25. https:// doi.org/10.1194/jlr.R051904

28. Song ZK, Wu HD, Cao HY, Qin L. The association between the LPA gene polymorphism and coronary artery disease in Chinese Han population. 2014;2014:370670. https://doi.org/10.1155/2014/370670.

29. McConnell JP, Guadagno PA, Dayspring TD, Hoefner DM, Thiselton DL, Warnick GR, Harris WS. Lipoprotein(a) mass: a massively misunderstood metric. J Clin Lipidol. 2014;8:550-3. https://doi.org/10.1016/j.jacl.2014.08.003.

30. Kamstrup PR, Nordestgaard BG. Elevated lipoprotein(a) levels, LPA risk genotypes, and increased risk of heart failure in the general population. JACC Heart Fail. 2016:4:78-87. https://doi.org/10.1016/j.jchf.2015.08.006

31. Paultre F, Pearson TA, Weil HF, Tuck CH, Myerson M, Rubin J, Francis CK, Marx HF, Philbin EF, Reed RG. High levels of Lp(a) with a small apo(a) isoform are associated with coronary artery disease in African American and white men. Arterioscler Thromb Vasc Biol. 2000;20:2619-24.

32. Li ZG, Li G, Zhou YL, Chen ZJ, Yang JQ, Zhang Y, Sun S, Zhong SL. Lack of association between lipoprotein(a) genetic variants and subsequent cardiovascular events in Chinese Han patients with coronary artery disease after percutaneous coronary intervention. Lipids Health Dis. 2013;12:127. https://doi.org/10.1186/1476-511x-12-127.

33. Lanktree MB, Rajakumar C, Brunt JH, Koschinsky ML, Connelly PW, Hegele RA. Determination of lipoprotein(a) kringle repeat number from genomic DNA: copy number variation genotyping using qPCR. J Lipid Res. 2009;50: 768-72. https://doi.org/10.1194/jlr.D800050-JLR200.

34. Sticchi E, Magi A, Kamstrup PR, Marcucci R, Prisco D, Martinelli I, Mannucci PM, Abbate R, Giusti B. Apolipoprotein(a) Kringle-IV type 2 copy number variation is associated with venous thromboembolism. PLoS One. 2016;11: e0149427. https://doi.org/10.1371/journal.pone.0149427.

Ready to submit your research? Choose BMC and benefit from:

- fast, convenient online submission

- thorough peer review by experienced researchers in your field

- rapid publication on acceptance

- support for research data, including large and complex data types

- gold Open Access which fosters wider collaboration and increased citations - maximum visibility for your research: over $100 \mathrm{M}$ website views per year

At BMC, research is always in progress.

Learn more biomedcentral.com/submissions 